\title{
Adaptive Iterative Learning Control for a Class of Nonlinear Systems with Unknown Control Directions
}

\author{
P. Jiang* and Y.Q. Cheng \\ School of Informatics, University of Bradford, $U K$
}

\begin{abstract}
An adaptive iterative learning control(ILC) approach is proposed for a class of uncertain nonlinear systems without prior knowledge about system control directions. The Nussbaum-type gain and the positive definite discrete matrix kernel are proposed for dealing with selection of the unknown control gain and learning of the repeatable uncertainties, respectively. Asymptotic convergence for a trajectory tracking within a finite time interval is achieved through repetitive tracking. Simulations are carried out to show the validity of the proposed control method.
\end{abstract}

\section{INTRODUCTION}

Iterative learning control(ILC) can deal with repeatable uncertainties through repetitive operations. Typical ILC's are designed based on the discrete Lyapunov method and the control output is updated in an affine fashion such as the $\mathrm{P}$ type or D type learning [1,2]. They require some preconditions on the learning gains. For example, given a linear dynamic system

$$
\begin{aligned}
& \left\{(t)=A x(t)+B u(t), x(t) \in R^{n}, x(0)=x_{0} \in R^{n},\right. \\
& y(t)=C x(t), y(t) \in R^{m}, u(t) \in R^{l},
\end{aligned}
$$

in order to guarantee convergence in terms of the $\lambda$-norm, the learning gain $L(t)$ should satisfy $\|I-C B L\|<1$. It implies that a priori knowledge about the control gain matrix $C B$ has to be available for learning control design. There exist other stability conditions, e.g., paper [3] proposed an adaptive high-gain iterative learning controller for a class of multiinput multi-output (MIMO) linear time-invariant systems but a priori knowledge about $C B$ was generally required, which should be positively definite. In some applications, such as uncalibrated visual servoing, it is difficult to obtain this kind of prior knowledge, that involves the external and internal parameters of a camera system, and the learning control may deteriorate to divergence due to control singularity [4]. In this case, an offline calibration process for parameter estimation or a trial run for gain tuning is necessary. The process is not only tedious but also requires a lot of human intervention with special expertise. In industry applications, there has been a tendency to develop techniques with less user intervention. Typically, products are engineered as systems to perform set functions. While they may work effectively from an engineering perspective, it is often at the expense of how the system will be used by real people [5]. A good product is one that not only works but is also easy to use even with less prior knowledge. In this context, it is necessary to study methodologies that are able to gain model knowledge for ILC parameter setting without user intervention if we expect that ILC's can be used by ordinary users in real applications.

*Address correspondence to this author at the School of Informatics, University of Bradford, UK; Tel: 00441274233940; Fax: 00441274236600; E-mail: P.jiang@Bradford.ac.uk
In adaptive control, the Nussbaum gain $[6,13]$ and the correction vector method [14] were proposed to deal with this kind of control problem without any prior model knowledge. For the purpose of robot trajectory imitation with an unknown camera-robot model, paper [15] proposed an indirect ILC for controlling systems without a priori knowledge. The indirect ILC identifies the gain matrix using a least square algorithm and then modifies the identified matrix, which might not yet converge to the true parameters, to avoid control singularity based on the correction vector method. Considering the fact that control of a system with less model knowledge involves two classes of learning that demand different resolutions, i.e. a coarse gain selection to satisfy the ILC preconditions and a fine leaning for compensating repeatable uncertainties, an ILC combining a Nussbaum gain incorporating with adaptive ILC's can be more suitable for real-time applications because it does not carry out explicit parameter identification. Nussbaum gain based ILC's have been studied in $[16,17]$ for single-input singleoutput (SISO) nonlinear systems with an unknown control direction. Designing an iterative learning controller using Nussbaum gain for MIMO systems is significant for its applications, e.g. for uncalibrated visual servoing that appears to be more difficult than SISO systems to gain model knowledge. Integrating with unmixing set, a Nusbaum gain based gain matrix selector was proposed for control of an MIMO linear system with nonsingular gain matrix [18].

This paper proposes an adaptive ILC for a class of minimum-phase MIMO nonlinear systems, where the repeatable nonlinear uncertainties are learned via an iterative learning law in a form of the positive definite discrete matrix kernel and the unknown control gain matrix, which is either positive definite or negative definite and is called a control direction, is dealt with by a Nussbaum gain for control direction probing. Under the control of the proposed algorithm, this paper shows that the unknown gain matrix is continuously probed and the control performance can be gradually improved through repetitive operations. The tracking error sequence can be asymptotic to zero at the end. The influence of Nussbaum gain to the control performance is studied in simulations.

\section{PROBLEM FORMULATION}

In this paper, we consider a class of nonlinear systems 


$$
\begin{aligned}
& \{(t, i)=f(t, i)+B[Y(x(t, i)) \cdot a(t, i)+u(t, i)], \\
& y(t, i)=C x(t, i),
\end{aligned}
$$

where $t$ denotes the time horizon; $i$ denotes the $i^{\text {th }}$ repetitive tracking; $B \in R^{n \times m}$ and $C \in R^{m \times n}$ are unknown constant input and output matrices, respectively; $Y(\cdot) \in R^{m \times p}$ is a measurable nonlinear matrix; $f(t, i) \in R^{n}$ and $a(t, i) \in R^{p}$ are unknown but repeatable vectors, i.e. $f(t, i)=f(t)$ and $a(t, i)=a(t) ; x(t, i) \in R^{n}, u(t, i) \in R^{m}$ and $y(t, i) \in R^{m}$ are the state, the control input and the control output at instant $t$ in the $i^{\text {th }}$ tracking, respectively.

Given a desired trajectories $r(t) \in R^{m}$ over a finite time interval $\left[0, T_{f}\right]$, the control objective is to design an iterative learning control $u(t, i)$ with the ability to reduce tracking errors for the whole trajectory in the time interval $\left[0, T_{f}\right]$ based on the past tracking experience, such that, as $i \rightarrow \infty$, the system tracking error $e(t, i)=y(t, i)-r(t) \rightarrow 0, \quad t \in\left[0, T_{f}\right]$. The follows are the assumptions of system (2):

Assumption 1. The control gain matrix $C B$ is symmetric and has spectrum $\sigma(C B)$ lying in either the open left $\left(C^{-}\right)$ or the open right $\left(C^{+}\right)$half complex plane.

Assumption 2. For every trial, the initial states can be reset to the desired states, i.e. $x_{i}(0)=x_{d}(0)=x^{0}, \forall i$.

The assumption 1 assumes that $C B$ is either positive definite or negative definite but the designer has no prior knowledge about the control direction. The assumption 2 is the initial resetting condition of ILC's.

\section{DESIGN OF CONTROL LAW}

For system (2), we know that

$$
f(t, i)=C f(t, i)+C B[Y(x(t, i)) \cdot a(t, i)+u(t, i)]
$$

The error equation can then be written as:

$$
\begin{aligned}
& \&(t, i)=C f(t, i)+C B[Y(x(t, i)) \cdot a(t, i)+u(t, i)]-(t) \\
& \left.=(C B)[C B)^{-1}(C f(t, i)-(t))+Y(x(t, i)) \cdot a(t, i)+u(t, i)\right]
\end{aligned}
$$

Notice that $(C B)^{-1}(C f(t, i)-\delta(t))$ is repeatable, i.e. it is invariant over repetitive index $i$, because of $f(t, i)=f(t)$. In addition, $a(t)=a(t, i)$ is repeatable too. Let $b(t)=(C B)^{-1}(C f(t)-f(t))$, the error equation can be rewritten with regard to two unknown but repeatable uncertainties, $a(t)$ and $b(t)$ :

$$
\&(t, i)=(C B)[b(t)+Y(x(t, i)) \cdot a(t)+u(t, i)]
$$

If we know that $C B$ is positive definite, an adaptive ILC can be proposed:

$$
u(t, i)=g_{l}(t, i)+g_{f}(t, i),
$$

where $g_{l}(t, i)=\hat{b}(t, i)+Y(x(t, i)) \hat{a}(t, i)$ is an adaptive iterative learning term for compensating the repetitive uncertainties and $g_{f}(t, i)=K e(t, i), K>0$, is a linear feedback term to cope with unrepeatable disturbances.
If we do not have the prior knowledge about $C B$, a coarse exploration of the gain direction must be carried out. In the paradigm of adaptive control, the Nussbaum gains were proposed for the exploration based on observation of a performance index [6,7]. A Nussbaum gain can be considered as a control-direction selector that can swing from positive to negative according to control performance, e.g. $h(y)=\cos \left(\frac{1}{2} \pi \xi\right) \exp \left(\xi^{2}\right), \cos (\xi) \exp \left(\xi^{2}\right), \xi^{2} \cdot \cos (\xi)$, and $\ln (\xi) \cdot \cos (\operatorname{sqrt}(\ln (\xi)))$, etc. Namely, a poor control performance, corresponding to a bigger $d \xi / d t$, tends to change the control gain to its opposite direction but a good control performance ceases this change. A Nussbaum gain has an increased amplitude due to the fixed probing period in the Nussbaum functions. It gives a chance to the system to correct deviation caused by the previous control using a wrong gain. This may cause poor transients during the process of probing, which are the expense of exploration and may happen in any trial run for gaining unknown knowledge, e.g. movement excitation for identification, human learning to back a car. Notice that this does not mean the control gain may always increase. Because probing of each direction using a Nussbaum gain has a whole sine curve, the gain could be stabilised at a suitable value depending on the current tracking performance.

Based on the above analysis, an adaptive ILC can be proposed for coping with unknown control direction:

$$
u(t, i)=v(k(t, i)) g(t, i)
$$

where $g(t, i)=g_{l}(t, i)+g_{f}(t, i)$ and $v(k(t, i))$ is a Nussbaumtype function with

$$
\begin{aligned}
& k\left(f_{t}, i\right)=g^{T}(t, i) e(t, i) \\
& k(0, i+1)=k\left(T_{f}, i\right), k(0,0)=0 .
\end{aligned}
$$

The control (5) integrates adaptive ILC, $g(t, i)$, with automatic gain selection using a Nussbaum gain, $v(k(t, i))$. In this paper, we choose a Nussbaum-type function of $v(\xi):=k^{2} \cos (k), k \in R$, which has the following property:

Property 1. [6] The Nussbaum-type function $v(\cdot)$ has the properties

$$
\begin{aligned}
& \limsup _{s \rightarrow \infty} \frac{1}{s} \int_{0}^{s} v(\xi) d \xi=+\infty, \\
& \liminf _{s \rightarrow \infty} \frac{1}{s} \int_{0}^{s} v(\xi) d \xi=-\infty .
\end{aligned}
$$

Remark 1: In the Nussbaum gain based control, the performance index was often set as a norm of control errors, e.g. $\mathcal{k}(t, i)=\|e(t, i)\|^{p}$. In this case, noise may cause increase in $k(t, i)$ and further result in a high control gain $v(k(t, i))$. However, in (6), the performance observation of learning control is taken as $g^{T}(t, i) e(t, i)$ that is the projection of the learned term onto the control error and reflects convergence of a learning process. It could exhibit robustness to measurement noise, which will be verified in the simulations. 
The control $g(t, i)$ in (5) consists of a learning control term $g_{l}(t, i)$ and a feedback control term $g_{f}(t, i)$ :

$$
g(t, i)=g_{l}(t, i)+g_{f}(t, i),
$$

with

$$
\begin{aligned}
& \left\{\begin{array}{c}
g_{l}(t, i)=\hat{b}(t, i)+Y(x(t, i)) \hat{a}(t, i) \\
\hat{b}(t, i)=\sum_{q=0}^{i} F_{1}(i-q) e(t, q) \\
\hat{a}(t, i)=\sum_{q=0}^{i} F_{2}(i-q) Y^{T}(x(t, q)) e(t, q)
\end{array}\right. \\
& g_{f}(t, i)=K e(t, i)
\end{aligned}
$$

where $F_{m}(\cdot), m=1,2$ are any positive definite discrete matrix kernels; $K$ is a positive constant.

The learning control (11) in a form of positive definite discrete matrix kernels is motivated by the discrete model reference adaptive system design using the hyperstability approach [19], where a sub-problem is to find the most general solutions for $\phi_{1}$ such that the following inequality holds:

$$
\begin{aligned}
& \sum_{k=0}^{k_{1}} v^{T}(k+1) w(k+1) \geq-\gamma_{0}{ }^{2}, \forall k_{1}>0 \\
& \text { with } w(k+1)=\left(\sum_{l=0}^{k} \phi_{1}(v, k, l)+A_{0}\right) y(k)
\end{aligned}
$$

where $\gamma_{0}{ }^{2}$ is an arbitrary positive finite constant; $v$ and $w$ are the $\mathrm{n}$-dimensional input and output of a block; $\phi_{1} \in R^{n \times q}$ represents the adaptive mechanism; $y$ is a finite qdimensional vector and $A_{0} \in R^{n \times q}$ is an unknown but constant matrix.

Property 2. [18] The inequality of (13) is satisfied by:

$$
\phi_{1}(v, k, l)=F(k-l) v(l+1)[G y(l)]^{T}
$$

where $F(k-l)$ is a positive definite discrete matrix kernel whose $Z$-transformation is a positive real discrete transfer matrix with a pole at $z=1$, and $G$ is a positive definite matrix.

Although the inequality (13) can be held only for any unknown constant matrix $A_{0}$, the property 2 is able to be extended as a general solution of ILC's in positive definite discrete matrix kernels for repeatable uncertainties, e.g. $a(i, t)=a(t)$, if the learning is conducted along the iterative horizon $i$. However, the adaptive controls in time-horizon, which are able to adapt the constant or slow time-varying uncertainties, e.g. $A_{0}$ in (13), are not very suitable for systems like (2), where $f(t, i)$ and $a(t, i)$ are both time-varying but repeatable. ILC can be an effective alternative.

If let $n(k)=v(k+1), a(t)=-A_{0}, y(k)=1$, and $G=1$ in Property 2 , we can obtain the following property along iterative horizon $i$ :

Property 3. [4] For any vector $n(i)$ and any repeatable constant vector $a(t)$, a positive definite discrete matrix kernel $F(i-q)$ ensures that the following accumulation along iterative horizon $i$ is always upper bounded:

$$
\sum_{i=0}^{N} n^{T}(i)\left(a-\sum_{q=0}^{i} F(i-q) n(q)\right) \leq \gamma_{0}{ }^{2}, \forall N
$$

where $\gamma_{0}^{2}$ is any positive finite constant.

Example: Similar to the Integral Adaptation in the Model Reference Adaptive Systems[18], $F(i)$ is taken as a diagonal matrix with elements of $\beta$ 's, $\beta>0$. It is obvious that $F(i)$ 's ztransformation $F(z)=\beta \cdot \operatorname{diag}\left(\frac{z}{z-1}, \mathrm{~L}, \frac{z}{z-1}\right)$ with a pole at $\mathrm{z}=1 . F(i)$ is a positive definite discrete matrix kernel because its $z$-transformation is a positive real discrete transfer matrix. This can be verified by:

(1) $F(z)$ is analytic outside the unit circle. The pole on the unit circle is simple and the associated residue is positive.

(2) Applying the transformation $z=(1+j \omega) /(1-j \omega)$ to $F(z)$ , its real part is given by

$$
\operatorname{Re}(F(j \omega))=\beta \cdot \operatorname{diag}\left(\frac{1}{2}, \mathrm{~L}, \frac{1}{2}\right)
$$

Therefore, for all real $\omega, \operatorname{Re}(F(j \omega))>0$.

Now, we are going to verify the boundedness of the accumulation for $F(i)=\operatorname{diag}(\beta, \ldots, \beta)$ :

$$
\sum_{i=0}^{N} n^{T}(i)\left(a-\sum_{q=0}^{i} F(i-q) n(q)\right)=\sum_{i=0}^{N} n^{T}(i)\left(a-\beta \sum_{q=0}^{i} n(q)\right)
$$

Let $\alpha=a / \beta$

$$
\begin{aligned}
& \sum_{i=0}^{N} n^{T}(i)\left(a-\sum_{q=0}^{i} F(i-q) n(q)\right)=\beta \sum_{i=0}^{N} n^{T}(i)\left(\alpha-\sum_{q=0}^{i} n(q)\right) \\
& =\beta\left[\begin{array}{l}
-n^{T}(0) n(0)-n^{T}(1) n(0)-n^{T}(1) n(1)-\mathrm{L} \\
-n^{T}(N) n(0)-n^{T}(N) n(1)-\mathrm{L}-n^{T}(N) n(N) \\
+n^{T}(0) \alpha+\mathrm{L}+n^{T}(N) \alpha
\end{array}\right] \\
& =\beta\left[\begin{array}{l}
-\frac{1}{2}\left(\sum_{i=0}^{N} n^{T}(q)-\alpha^{T}\right)\left(\sum_{i=0}^{N} n(q)-\alpha\right)-\frac{1}{2} \sum_{i=0}^{N} n^{T}(q) n(q) \\
+\frac{1}{2} \alpha^{T} \alpha
\end{array}\right] \\
& \leq \frac{1}{2} \beta \alpha^{T} \alpha=\gamma_{0}^{2}
\end{aligned}
$$

Remark 2: In the proposed ILC (11), if $F_{1}$ and $F_{2}$ are selected to be $\operatorname{diag}\left(\beta_{1} \ldots, \beta_{1}\right)$ and $\operatorname{diag}\left(\beta_{2} \ldots, \beta_{2}\right), \beta_{1}>0$ and $\beta_{2}>0$, they lead to a P-type feedback learning using current tracking errors:

$$
\begin{aligned}
& \hat{b}(t, i)=\sum_{q=0}^{i} F_{1}(i-q) e(t, q)=\hat{b}(t, i-1)+\beta_{1} e(t, q) \\
& \hat{a}(t, i)=\sum_{q=0}^{i} F_{2}(i-q) Y^{T}(x(t, q)) e(t, q) \\
& =\hat{a}(t, i-1)+\beta_{2} Y^{T}(x(t, q)) e(t, q)
\end{aligned}
$$

Classical ILC's use only the previous tracking errors for current learning [20]. It has an advantage that feedforward compensation can be calculated offline and be implemented online through a simple mechanism of retrieval-frommemory. Some researchers introduced current iterative track- 
ing error into learning, called feedback ILC, and argued that could improve robustness to uncertainties, the tracking error bound and the ILC convergence rate $[21,22]$. The discrete definite positive kernels can provide a general class of feedback ILC's.

Remark 3: Other ILC's can be derived from various positive definite discrete matrix kernels, e.g. an ILC in a form of a low pass filter along repetitive index:

$$
\left\{\begin{array}{c}
W(t, i)=\sigma W(t, i-1)+(1-\sigma) e(t, i) \\
\hat{b}(t, i)=\hat{b}(t, i-1)+W(t, i)
\end{array} .\right.
$$

where the coefficient $\sigma$ of the filter must be less than $1 / 3$ in order to be a positive definite discrete matrix kernel.

It will be proved that the adaptive ILC of (5) that integrates the adaptive ILC in discrete positive definite matrix kernels with the Nussbaum gain can cope with repeatable uncertainties and together with an unknown gain matrix.

Theorem 1. Under the two assumptions and the control (5), $k(t, i)$ is bounded and the repetitive tracking error sequence is asymptotic to zero when iterations go to infinity, i.e. $\|e(t, i)\| \rightarrow 0$ as $i \rightarrow \infty$.

Proof of Theorem 1:

By Assumption 1 there must exist an $\beta \in\{-1,1\}$ such that $\beta C B$ is symmetrically and positively definite. We define $Q:=(\beta C B)^{-1}=\beta(C B)^{-1}$, then $Q$ is symmetrically and positively definite too and $Q C B=\beta I$. We define a Lyapunov equation:

$$
V(t, i):=\frac{1}{2}<e(t, i), Q e(t, i)>, t \in\left[0, T_{f}\right] .
$$

From (3) and (10), we have

$$
\begin{aligned}
& \{(t, i)=<Q e(t, i), \dot{e}(t, i)> \\
& =<Q e(t, i),(C B)[b(t)+Y(x(t, i)) a(t)+u(t, i)]> \\
& =<e(t, i), \beta b(t)+\beta Y(x(t, i)) a(t)-\hat{b}(t, i)-Y(x(t, i)) \hat{a}(t, i) \\
& -g_{f}(t, i)+g(t, i)+\beta u(t, i)>
\end{aligned}
$$

Because of Assumption 2, i.e. $V(0, i)=0$, the total energy of the repetitive tracking from $(0,0)$ to $(t, i)$ can be obtained by:

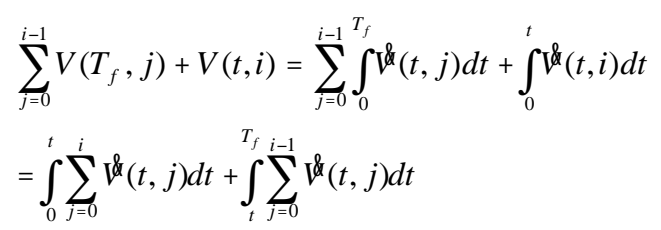

Substituting (14) into it, we have

$$
\begin{aligned}
& \sum_{j=0}^{i-1} V\left(T_{f}, j\right)+V(t, i) \\
& =\int_{0}^{t} \sum_{j=0}^{i}<e(t, j), \beta b(t)-\hat{b}(t, j)>d t+ \\
& \int_{t}^{T_{f}} \sum_{j=0}^{i-1}<e(t, j), \beta b(t)-\hat{b}(t, j)>d t+
\end{aligned}
$$

$$
\begin{aligned}
& \int_{0}^{t} \sum_{j=0}^{i}<e(t, j), Y(x(t, j))[\beta a(t)-\hat{a}(t, j)]>d t+ \\
& \int_{t}^{T_{f}} \sum_{j=0}^{i-1}<e(t, j), Y(x(t, j))[\beta a(t)-\hat{a}(t, j)]>d t+ \\
& \sum_{j=0}^{i-1} \int_{0}^{T_{f}}<e(t, j),-g_{f}(t, j)>d t+\int_{0}^{t}<e(t, i),-g_{f}(t, i)>d t+ \\
& \sum_{j=0}^{i-1} \int_{0}^{T_{f}}<e(t, j), \beta u(t, j)+g(t, j)>d t \\
& +\int_{0}^{t}<e(t, i), \beta u(t, i)+g(t, i)>d t
\end{aligned}
$$

From the property 3 of a positive definite discrete matrix kernel, the updating laws of $\hat{b}(t, i)$ and $\hat{a}(t, i)$ along iterative horizon can guarantee upper-boundedness of the first four terms, i.e.:

$$
\begin{aligned}
& 0 \leq \sum_{j=0}^{i-1} V\left(T_{f}, j\right)+V(t, i) \\
& \leq 2 \int_{0}^{T_{f}} \max \left(\gamma_{1}^{2}, \gamma_{2}^{2}, \gamma_{3}^{2}, \gamma_{4}^{2}\right) d t- \\
& \sum_{j=0}^{i-1} \int_{0}^{T_{f}} K e^{T}(t, j) e(t, j) d t-\int_{0}^{t} K e^{T}(t, i) e(t, i) d t+ \\
& \sum_{j=0}^{i-1} \int_{0}^{T_{f}}(\beta v(k)+1) g^{T}(t, j) e(t, j) d t \\
& +\int_{0}^{t}(\beta v(k)+1) g^{T}(t, i) e(t, i) d t \\
& \leq 2 \gamma^{2} \cdot T_{f}+\sum_{j=0}^{i-1} \int_{k(0, j)}^{k\left(T_{f}, j\right)}(\beta v(k)+1) d k+\int_{k(0, i)}^{k(t, i)}(\beta v(k)+1) d k \\
& =2 \gamma^{2} \cdot T_{f}+\int_{k(0,0)}^{k(t, i)}(\beta v(k)+1) d k
\end{aligned}
$$

where $\gamma^{2}=\max \left(\gamma_{m}{ }^{2}\right), m=1,2,3,4$, is a positive finite constant and equation (6) and equation (7) have been applied. Thus

$$
\int_{k(0,0)}^{k(t, i)}(\beta v(k)+1) d k \geq-2 \gamma^{2} \cdot T_{f}
$$

which is lower bounded. It can be rewritten as

$$
\int_{k(0,0)}^{k(t, i)} \beta v(k) d k \geq-(k(t, i)-k(0,0))-2 \gamma^{2} \cdot T_{f}
$$

Suppose, at any time instant, $k(t, i)$ becomes divergent. We consider the cases of positive and negative divergence:

1) $k(t, i) \rightarrow+\infty$

Equation (17) implies $\lim _{k(t, i) \rightarrow+\infty} \frac{1}{k(t, i)} \int_{k(0,0)}^{k(t, i)} \beta v(k) d k \geq-1$, where $k(0,0)=0$. It contradicts (9) if $\beta$ is +1 or contradicts (8) if $\beta$ is -1 ;

2) $k(t, i) \rightarrow-\infty$ 
Equation (17) implies $\lim _{k(t, i) \rightarrow-\infty} \frac{1}{k(t, i)} \int_{k(0,0)}^{k(t, i)} \beta v(k) d k \leq-1$, where $k(0,0)=0$. It contradicts (8) if $\beta$ is +1 or contradicts (9) if $\beta$ is -1 .

So $k(t, i)$ and thus $\int_{k(0,0)}^{k(t, i)}(\beta v(k)+1) d k$ must keep bounded for repetitive tracking. Then, from (15), we know that $\sum_{j=0}^{i-1} V\left(T_{f}, j\right)+V(t, i)$ is bounded as a result. For this positive and monotonic series, we know that $V(t, i) \rightarrow 0$ as $i \rightarrow \infty$, i.e. $\|e(t, i)\| \rightarrow 0, \forall t \in\left[0, T_{f}\right]$.

\section{NUMERICAL EXAMPLES}

Considering the following 2-dimensional system with an unknown gain matrix $B$ and a repeatable uncertainty $f(t)$ :

$$
\&=f(t)+B u
$$

where $f(t)=\left[\begin{array}{l}2 \sin (t) \\ 3 \cos (t)\end{array}\right]$ and $B=\left[\begin{array}{cc}1 & 0.5 \\ 0.5 & 1\end{array}\right]$ are supposed to be unknown.

Because of the time-varying uncertainty $f(t)$, adaptive control along time-horizon is not adequate for control of this sort of systems but ILC's can be an effective alternative.

Based on the Theorem 1, an adaptive ILC can be constructed for tracking control of a given trajectory:

$$
r(t)=\left[\begin{array}{c}
5 \cos (\pi-\pi \cdot t / 10)+5 \\
5 \sin (\pi-\pi \cdot t / 10)
\end{array}\right], t \in[0,5](s)
$$

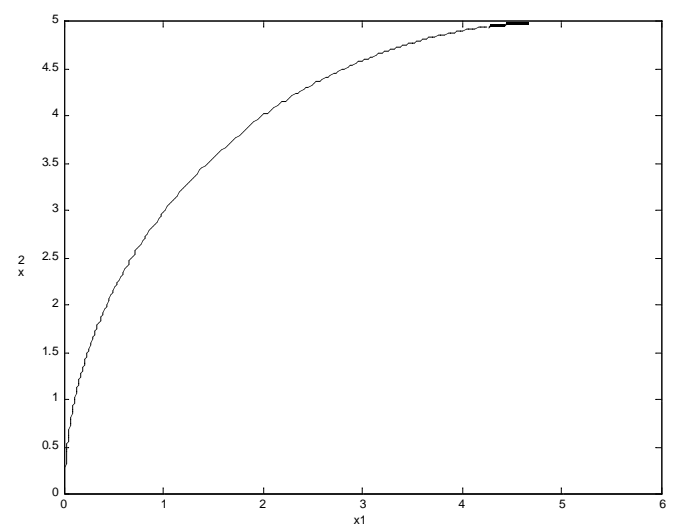

Fig. (1). The desired trajectory.

The control law is designed below with a sampling period of $20 \mathrm{~ms}$.

$$
u(t, i)=v(k(t, i))\left(\sum_{q=0}^{i} F_{1}(i-q) e_{m}(t, q)+K e_{m}(t, i)\right)
$$

where the discrete positive definite matrix kernel is set to be a positive definite matrix, i.e. $F_{1}()=.\operatorname{diag}(2,2)$, and the feedback gain $K=1.5$. The control (20) consists of a control direc- tion selector in a form of the Nussbaum functions and a history based learning control. In order to verify robustness of the Nussbaum gain, suppose that there exists a random measurement noise $n$ in uniform distribution on the interval of $[-0.05,0.05]$, i.e., $e_{m}(t, i)=e(t, i)+n$.

Fig. (2) depicts the first tracking errors. In the first tracking, because the controller did not know its correct control direction and did not have any compensation to the movement, it showed a big tracking error that caused the Nussbaum gain to probe the correct control direction. The evolution of the control performance observation $k(t, i)$ and the corresponding Nussbaum gain are plotted in Fig. (3). From the figure, we did not see any evidence of divergence due to the measurement noise and the coarse gain probing reached the correct gain after the first trial. The RMS(Root Mean Square) error of the iterative learning control is illustrated in Fig. (4). It clearly indicates the learning and control capabilities of the proposed control law that includes a coarse probing of the control direction and a fine tuning for movement and uncertainty compensation. The control errors of the $30^{\text {th }}$ tracking is depicted in Fig. (5). Comparing with the control errors of the $1^{\text {st }}$ tracking in Fig. (2), we can find that the maximum tracking errors in both directions are reduced from ex $1_{\max }=6.189$ and $e x 2_{\max }=11.05$ to $e x 1_{\max }=0.096$ and ex $2_{\max }=0.086$.

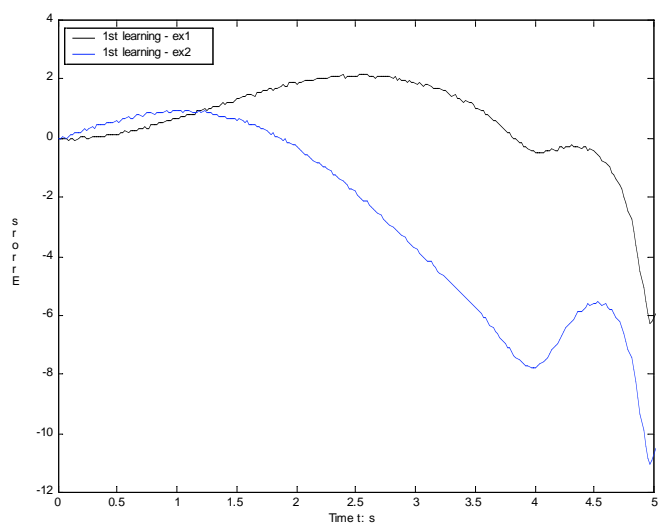

Fig. (2). The first tracking errors for the case 1.

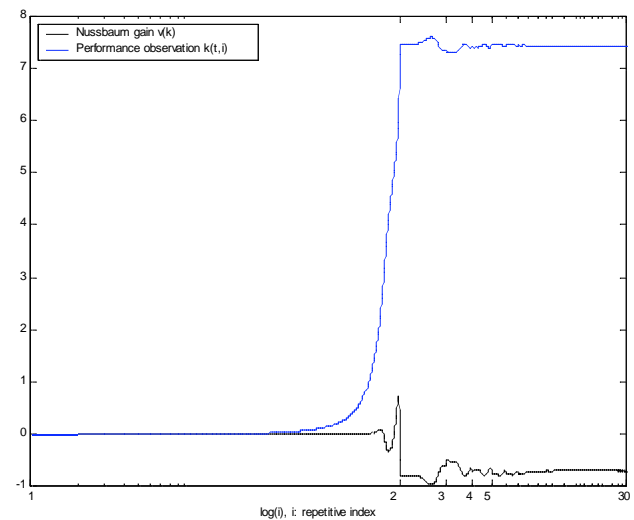

Fig. (3). Nussbaum gain $v$ and $k(t, i)$ for the case 1 . 


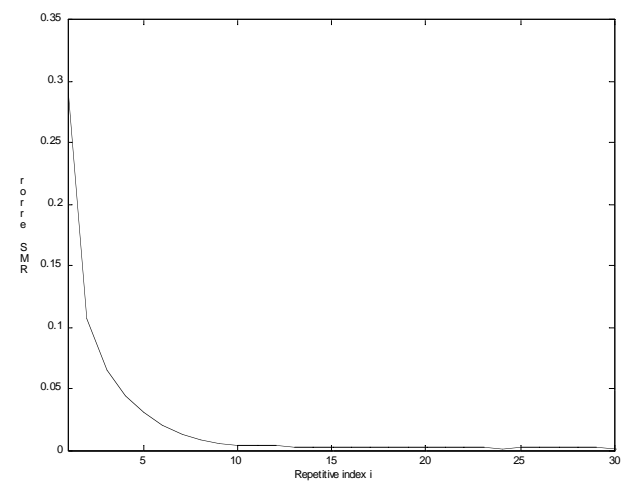

Fig. (4). RMS error of the ILC for the case 1.

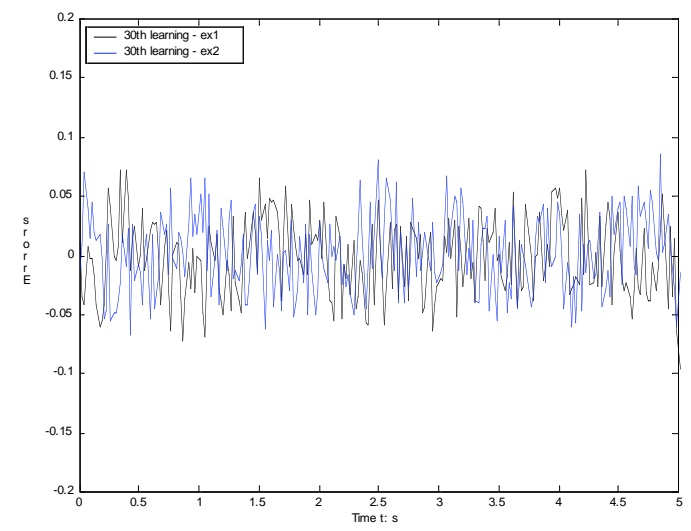

Fig. (5). The $30^{\text {th }}$ tracking errors for the case 1 .

Now we suppose an unknown system with a repeatable uncertainty of $f(t)=\left[\begin{array}{c}-0.5 e^{0.1 t} \\ e^{0.1 t}\end{array}\right]$ and $B=\left[\begin{array}{cc}-1 & 0.5 \\ 0.5 & -1\end{array}\right]$, which is negatively definite. The same control law is applied for the trajectory tracking. Fig. (6) and Fig. (7) show the $1^{\text {st }}$ and $30^{\text {th }}$ tracking errors. The proposed control law can automatically probe the control gain quickly as shown in Fig. (8).

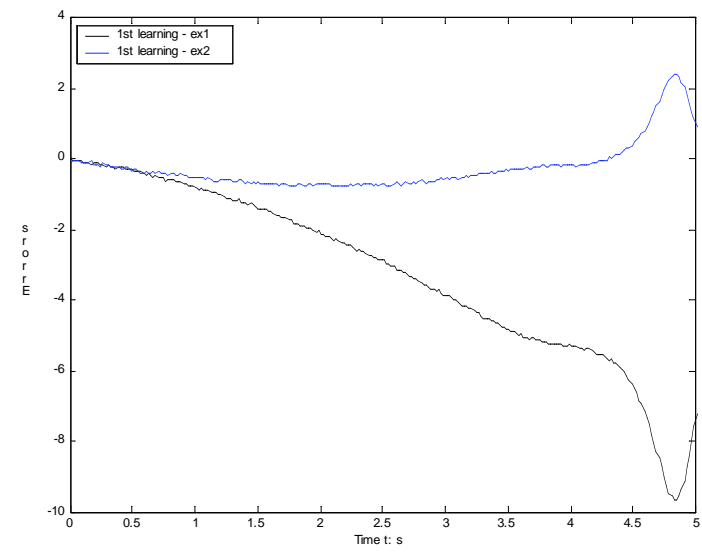

Fig. (6). The first tracking errors for the case 2 .

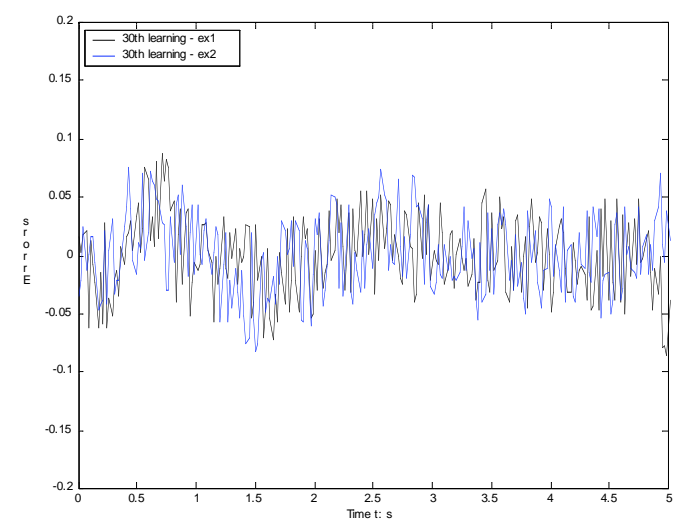

Fig. (7). The $30^{\text {th }}$ tracking errors for the case 2.

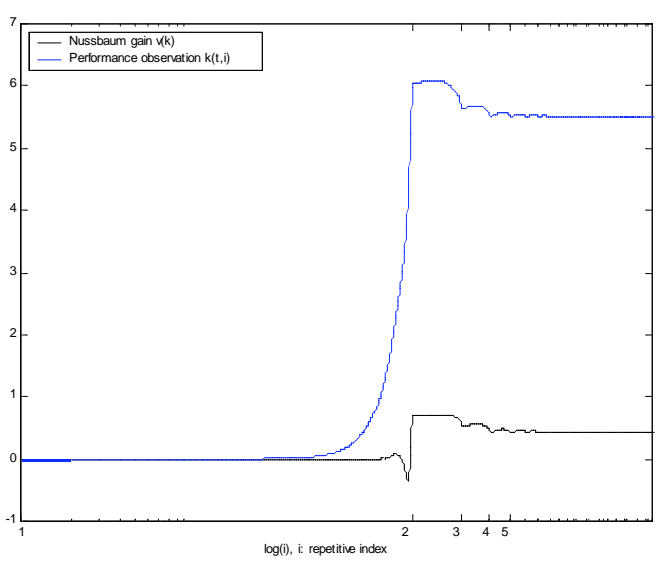

Fig. (8). Nussbaum gain $v$ and $k(t, i)$ for the case 2 .

Notice the evolution of the Nussbaum gain in Fig. (3) and Fig. (8), we can make the following conclusions:

1) the Nussbaum gain can find out the correct gain rapidly within the first trial and thereafter tracking will contribute to the learning of compensation as typical ILC's;

2) an interesting phenomenon can be observed that steep increase of $k(t, i)$ and the corresponding Nussbaum gain $v$ usually occur near the end of the first tracking. It is caused by large tracking errors and exhibits more oscillation for gain exploration. However, after start of the second tracking, both $k(t, i)$ and $v$ are quickly stabilised because of the initial resetting of ILC's after a short period of local tunings. Consequently, repetitive resetting is a good strategy for Nussbaum function based gain selection, which gives a chance to do fine tuning of the gain. It also inspires us that, in the Nussbaum-gain based adaptive control, a reference rectifying strategy, i.e. modifies the desired trajectory to align with the current state and forces control error to zero regularly, could effectively help stabilise the gain-selection process and avoid extremely high-gain control, which also implies that a better transient performance could be achieved;

3 ) even with a measurement noise in a range of [$0.05,0.05]$, both $k(t, i)$ and the Nussbaum gain $v(k(t, i))$ can be stabilised. 


\section{CONCLUSIONS}

Integrating Nussbaum gains with positive definite discrete matrix kernels, this paper proposed an adaptive ILC consisting of a coarse control direction selector and a fine learning control for a class of nonlinear systems. The systems may include time-varying but repeatable linear parameters and an unknown positive or negative definite control gain matrix. Without any prior knowledge about these uncertainties, the paper proved that the output is able to converge to the desired one through repetitive tracking. The simulations showed that Nussbaum gains can effectively incorporate with adaptive ILC's to control a system with an unknown control direction.

\section{REFERENCES}

[1] G. Heinzinger, D. Fenwick, B. Paden and F. Miyazaki, "Stability of learning control with disturbances and uncertain initial condition", IEEE Trans. Automat. Control, 37: 110-114, 1992.

[2] S.S. Saab, "On the P-type learning control", IEEE Trans. Automat. Control, 39: 2298-2302, 1994.

[3] D. H. Owens and G. Munde, "Stability of a multi-input, multioutput adaptive iterative learning control system", European Control Conference, Brussels, July 1997: TU-E B6.

[4] P. Jiang and R. Unbehauen, "Robot visual servoing with iterative learning control", IEEE Trans. Syst. Man, Cybern-Part A: Syst. Hum., 32(2): 281-287, 2002.

[5] J. Preece, Y. Rogers and H. Sharp, Interaction Design, John Wiley \& Sons, Inc., 2002.

[6] R.D. Nussbaum, "Some remarks on the conjecture in parameter adaptive control", Syst. Control Lett., 3: 243-246, 1983.

[7] B. Martensson, "Remarks on adaptive stabilization of first order nonlinear systems", Syst. Control Lett., 14: 1-7, 1990.

[8] E.P. Ryan, "A universal adaptive stabilizer for a class of nonlinear systems", Syst. Control Lett., 16: 209-218, 1991.
[9] E.P. Ryan, "Universal $W^{1, \infty}$-tracking for a class of nonlinear systems", Syst. Control Lett., 18: 201-210, 1992.

[10] E.P. Ryan, "A nonlinear universal servo mechanism", IEEE Trans. Automat. Control, 39: 753-761, 1994.

[11] M. Corless and E.P. Ryan, "Adaptive control of a class of nonlinearly perturbed linear systems of relative degree two", Syst. Control Lett., 21: 59-64, 1993.

[12] A. Ilchmann and E.P. Ryan, "Universal $\lambda$-tracking for nonlinearly perturbed systems in the presence of noise", Automatica, 30: 337346, 1994.

[13] X.D. Ye and J.P. Jiang, "Adaptive nonlinear design without a priori knowledge of control directions", IEEE Trans. Automat. Control, 43: 1617-1621, 1998.

[14] R. Lozano, "Singular-free adaptive pole-placement without resorting to persistency of excitation: detailed analysis for first order systems", Automatica, 28(1): 27-33, 1992.

[15] P. Jiang, L.C. Bamforth, Z.R. Feng, J.E. Baruch and Y.Q. Chen, "Indirect iterative learning control for a discrete visual servo without a camera-robot model", IEEE Trans. Syst. Man, Cybern.Part B: Cybernetics, 37(4): 863-876, 2007.

[16] H.D. Chen and P. Jiang, "Adaptive iterative learning control for nonlinear systems with unknown control", ASME J. Dyn. Syst. Meas. Control, 126: 916-920, 2004.

[17] J.X. Xu and R. Yan, "Iterative learning control design without a priori knowledge of the control direction", Automatica, 40: 18031809, 2004.

[18] P. Jiang, H.D. Chen, L.C. Bamforth, "A universal iterative learning stabilizer for a class of MIMO systems", Automatica, 42(6): 973981, 2006.

[19] Y.D. Landau, Adaptive Control-the Model Reference Approach, New York: Marcel Dekker, 1979.

[20] K. Moore, "Iterative learning control-an expository overview", Appl. Comput. Controls, Signal Process Circuit., 1: 425-288, 1998.

[21] M. Sun and D. Wang, "Closed-loop iterative learning control for non-linear systems with initial shifts", Int. J. Adapt. Control Signal Process, 16: 515-538, 2002.

[22] M. French and E. Rogers, "Nonlinear iterative learning by an adaptive Lyapunov technique", Int. J. Control, 73(10): 840-850, 2000.

(C) Jiang and Cheng; Licensee Bentham Open.

This is an open access article distributed under the terms of the Creative Commons Attribution License (http://creativecommons.org/license/by/2.5/), which permits unrestrictive use, distribution, and reproduction in any medium, provided the original work is properly cited. 